\title{
Modified multiple drug therapy in the National Leprosy Eradication Programme, India
}

\author{
A C McDOUGALL \\ Department of Dermatology, The Slade Hospital, Headington, \\ Oxford $\mathrm{OX} 37 \mathrm{JH}$, England
}

Accepted for publication 23 April 1992

The National Leprosy Control Programme (NLCP) started in India in 1955, using dapsone monotherapy, with emphasis on health education, survey, case detection and rehabilitation. The Central Government changed its strategy and name to the National Leprosy Eradication Programme (NLEP) in 1982-83, following the recommendations of the Swaminathan Committee. ' Since 1983 multiple drug therapy (MDT), essentially as recommended by the World Health Organization (WHO) in $1982,{ }^{2}$ but with an intensive phase of daily medication for 2 weeks for multibacillary cases, has been implemented in a phased manner, with emphasis on high endemic districts. By 1990, it was possible to report that 1.6 million out of the 2.4 million registered patients were under MDT and that the number of cases discharged from treatment was exceeding the number of new cases detected each year. ${ }^{3}$ About 4.5 million cases have been taken off the leprosy registers in India during the past 10 years.

Despite this evidence of progress, an appraisal of the situation in India in early 1990 indicated that the pace and extent of the implementation of MDT was unsatisfactory. From the previously identified total of 196 districts with a prevalence of 5 or more per 1000 of the population, 66 had been unable to implement MDT, mainly because of an inadequate NLEP infrastructure, coupled with poor prospects for the recruitment and training of staff in the foreseeable future. Professor G K Vishwakarma, Director of Health Services, undertook a critical review of the situation (which was likely, if not tackled, to undermine the objective of eradication of leprosy in India by the year 2000), and this led the formulation of a 'modified' MDT approach, later published as Guidelines for Modified MDT Scheme in Selected Districts by the Leprosy Division of the Ministry of Health and Family Welfare, New Delhi. ${ }^{4}$ The Foreword draws attention to the main concern of bringing the remaining 66 districts under MDT as soon as reasonably possible, using the primary health care (PHC) infrastructure (as opposed to the vertical, specialized structure of the NLEP), and to the main objective of the 66-page booklet in providing guidelines to all categories of health staff for the extension of MDT through, '... an economically feasible system of service delivery'.

* Correspondence: 87 Lower Radley, Near Abingdon, Oxfordshire OX14 3BA, England. 
The methodology of modified MDT (MMDT) is described in considerable detail; there are 18 main sections, 6 annexures and 17 appendices. The salient features are described under two headings-administrative and technical. The former includes instructions for the setting up of a District Leprosy Society, under the chairmanship of the District Collector, which is to take overall responsibility for the operation of the programme. The District or Zonal Officer of the District will act as secretary and the Chief Medical Officer of the District as vice-chairman. A system of awards or incentives is described for: a, PHC functionaries, based on performance, together with the sum of 3 rupees for each new case detected; and b, patients, who will receive 10 rupees for each supervised monthly dose and 100 rupees if and when they complete the prescribed course of MMDT for either pauci- or multibacillary leprosy. The technical directions are 4 in number: 1 , the period of initial, intensive chemotherapy, as currently used in the NLEP, ${ }^{5}$ giving dapsone $100 \mathrm{mg}$, clofazimine $100 \mathrm{mg}$ and rifampicin $600 \mathrm{mg}$ daily for the first 14 days, followed by the standard WHO regimen, will not be used; 2, fixed duration chemotherapy ( 2 years for multi- and 6 months for paucibacillary cases) will be the rule. Thus multibacillary cases no longer need to continue treatment ' . . f for a period of 2 years (24 months) or until achievement of smear negativity, whichever is later' ${ }^{5}$ However the Guidelines include provision for the continuation of treatment in certain paucibacillary cases, beyond 6 months, especially in cases with multiple lesions. This step is to be taken only af ter careful review by a medical officer, including detailed clinical and bacteriological examination for errors in classification; 3, the rapid survey approach described in existing NLEP guidelines will be replaced by intensification of health education activities to encourage voluntary reporting; and 4, the bacteriological examination of skin smears in MMDT programmes will be confined to multibacillary cases and suspected multibacillary cases. However, MMDT is to be started on the basis of clinical examination.

The annexures deal with administrative, technical and financial components in detail and there are clear instructions on the registers, records and reports needed. Furthermore, 2 training manuals, 1 for medical officers and health supervisors and the other for health workers, have recently been published by the Leprosy Division, specifically for the implementation of MMDT through existing health care services. ${ }^{6}$

MMDT is reported to have been started already, on a limited scale, in a few districts in India and a conference was held in January 1992 in Puri, Orissa, to discuss the steps needed for the implementation of MMDT in the 2 districts of Keonjhar and Kalahandi. This brought together representatives from the Directorate of Health Services for Orissa, the Gandhi Memorial Leprosy Foundation, DANLEP, The Damien Institute (Bhubaneswar), Cuttack Medical College and The Damien Foundation (Belgium). Solutions were sought to questions concerning the re-deployment of staff and their training in leprosy control, the working relationship between NLEP and PHC staff, the change from dapsone monotherapy (used for the better part of 20 years), to the effective use of MMDT, through the PHC system, the provision of transport specifically for leprosy work and the constant availability of adequate stocks of dapsone, clofazimine and rifampicin at all times. The meeting in Puri (possibly one of the first to systematically plan for the use of MMDT) emphasized the importance of preliminary screening of all registered 'cases' in order to clearly identify those in need of chemotherapy - a procedure which invariably results in marked reduction in the true prevalence figures.

Greatly inspired by Mrs Indira Gandhi's personal interest and support for leprosy 
work in the early 1980s, it is the stated intention of the Government of India to eradicate leprosy by the year 2000. Whether this may need revision to "elimination'7 or to "the provision of MDT for all by the year 2000' has yet to be seen, but meanwhile there is no doubt that the guidelines described above are evidence of the determination of the health authorities in India to radically improve the present situation without delay. They could well be studied by those responsible for programmes in other parts of the world (Brazil, Myanmar, Indonesia, Nigeria and several other African countries come to mind) where the benefits of MDT have as yet been made available to only a small percentage of those in need. Furthermore, in view of the many thousands of patients, in 66 districts of India, for whom MMDT has been designed, careful monitoring of this initiative may provide valuable information on the feasibility and effectiveness of using the PHC system, with main responsibility at district level.

\section{References}

1 National Leprosy Eradication Programme in India. Status Report 1985-86. Leprosy Division, Directorate General of Health Services, Ministry of Health and Family Welf are, Nirman Bhavan, New Delhi. 1986.

2 WHO. Chemotherapy of leprosy for control programmes. Report of a WHO Study Group. Technical Report Series 675. WHO, Geneva, 1982.

3 WHO. Report of the consultation on technical and operational aspects of leprosy. WHO/CTD/LEP/90.3. Limited distribution. WHO, Geneva, 1990.

4 National Leprosy Eradication Programme in India. Guidelines for Modified MDT Scheme in Selected Districts. Leprosy Division, New Delhi; as in reference 1. 1990.

5 National Leprosy Eradication Programme in India. Guidlines for Multidrug Treatment in Endemic Districts. Leprosy Division, New Delhi; as in reference 1. 1989.

6 National Leprosy Eradication Programme in India. Training manuals for (a) Medical Officers and Health Supervisors and (b) Health Workers. Leprosy Division, New Delhi; as in reference 1. 1990.

7 WHO. Towards elimination of leprosy. WHO/CTD/LEP/91.1. Leprosy Control Programme. WHO, Geneva, 1991. 